\title{
Pollution physico-chimique et microbiologique de l'eau des puits dans la Commune d'Abomey-Calavi au Bénin en 2009
}

\author{
C. DEGBEY ${ }^{1,3^{*}}$, M. MAKOUTODE ${ }^{2}$, E. M. OUENDO ${ }^{2}$ et C. DE BROUWER ${ }^{1}$ \\ ${ }^{1}$ Université Libre de Bruxelles, Unité de Santé au travail et de toxicologie du milieu, Ecole de Santé Publique, \\ Campus Erasme, CP-593, Route de Lennik, 808-B-1070 Bruxelles/ Belgique. \\ ${ }^{2}$ Université d'Abomey-Calavi, Institut Régional de Santé Publique (IRSP), Route des Esclaves, 01 BP 918 , \\ Cotonou /Bénin. \\ ${ }^{3}$ Unité de Santé au Travail et de Toxicologie du Milieu, Ecole de Santé Publique, Campus Erasme, CP-593, \\ Route de Lennik, 808-B-1070 Bruxelles/Belgique. \\ *Auteur correspondant ; E-mail : comlancy@yahoo.fr ; GSM: 0032493608902/0022995960541 ; \\ Tel : +32(0)2 5554027, FAX:+32(0)2 5554049.
}

\section{RESUME}

Une étude descriptive et analytique sur l'alimentation en eau de boisson a été réalisée sur une population de 110 puits dans la commune d'Abomey-Calavi au Bénin qui sont respectivement sélectionnés par tirage aléatoire simple dans l'ensemble des 55898 ménages de la commune. L'objectif de ce travail est d'évaluer le niveau de pollution des eaux de puits consommées par la population de la Commune et son lien avec la santé. La pollution physico-chimique et microbiologique des puits dans cette commune, ville semiurbaine de 307.745 habitants, a été étudiée. Les puits présentent une forte pollution en nitrites, en nitrates, en aluminium, en fer total, en cadmium et en mercure qui sont respectivement de 32,73\%, 11,82\%,10\%, 43,64\%, $6,36 \%$ et $14,55 \%$ d'une part et d'autre part, une pollution microbiologique (bactéries, parasites et champignons) qui rendent l'eau impropre à la consommation. L'origine des pollutions peut être attribuée à diverses causes: défaut d'assainissement et de collecte des ordures ménagères, transfert de polluants à partir des couches superficielles, conditions de puisage et structure des installations. À terme, l'utilisation des puits pourrait constituer un risque sanitaire important pour la majorité des habitants de la zone d'étude.

(c) 2010 International Formulae Group. All rights reserved.

Mots clés : risque sanitaire, assainissement, diarrhées, ville semi-urbaine, nappes souterraines, limite admissible.

\section{INTRODUCTION}

L'eau est une ressource essentielle. Son usage à des fins alimentaires, d'hygiène corporelle ou récréatives nécessite un haut niveau de qualité physico-chimique, chimique et microbiologique. Les substances qu'elle transporte sont en effet susceptibles d'être ingérées, inhalées, ou d'entrer en contact avec la peau.

Dans les pays en développement, jusqu'à $80 \%$ des maladies et plus du tiers des décès sont imputables aux maladies à transmission hydrique (OMS, 2003). La gastro-entérite est la maladie la plus 
fréquemment associée à l'ingestion d'eau contaminée par des matières fécales et entraîne des conséquences très graves sur la santé (Bourne et al., 2007). D'autres maladies plus rares comme les hépatites ou les méningites peuvent aussi être provoquées par l'ingestion d'eau contaminée. Au Bénin, selon les statistiques du Ministère de la Santé, les maladies d'origine hydrique représentent $54 \%$ de l'ensemble des maladies (Annuaire des statistiques sanitaires du Bénin, 2009). La prédominance des diarrhées est seulement en partie due au manque de disponibilité d'une source d'eau potable sûre et les facteurs sociaux tels que les mauvaises pratiques d'hygiène sont également mis enjeu (Pande et al., 2008). Dans le cadre de la prévention des maladies à transmission hydrique, la qualité et l'hygiène de l'eau sont des exigences prioritaires de santé publique. L'eau destinée à la consommation humaine ne doit contenir ni substances chimiques dangereuses, ni germes nocifs pour la santé (Kreamer et al., 2009). Dans la commune d'Abomey-Calavi, seule une partie des quartiers de la ville est desservi par le réseau d'eau potable. La majorité des ménages utilisent donc l'eau provenant des puits. Plus de $70 \%$ de la population de la commune d'Abomey-Calavi (307.745 habitants) est actuellement approvisionnée en eau par les puits et forages des nappes souterraines (PNE, 2006). Tous les ménages de la commune disposent d'un puits. La faible couverture en eau potable et les comportements à risque des populations sont à l'origine des maladies graves telles que les diarrhées, la fièvre typhoïde et paratyphoïde, la dysenterie amibienne. Ces facteurs ont favorisé l'apparition des différents épisodes de choléra que le Bénin a connu respectivement en 2006, 2007, 2008, 2009 (Annuaire des statistiques sanitaires du Bénin, 2009).

$\mathrm{Au}$ Bénin, les eaux souterraines protégées géologiquement sont exposées à des pollutions agricole, industrielle ou urbaine. La croissance démographique est un indicateur important de la demande en eau potable. Cette situation engendre de nombreux problèmes dont les plus importants sont: la pénurie d'eau de bonne qualité, la dégradation de la qualité de l'eau et la détérioration de la santé. La nature hydrogéologique de la commune d'Abomey-Calavi présente une perméabilité élevée du sol, ce qui favorise une pollution assez rapide de la nappe phréatique avec un faible pouvoir de filtration. Cette commune connaît par moment le phénomène d'inondation dont la conséquence est l'infiltration de l'eau de ruissellement qui peut contaminer l'eau des puits.

L'objectif de cette étude est de déterminer les caractéristiques microbiologiques et physico-chimiques de l'eau de boisson dans la commune d'Abomey-Calavi au Bénin. Un suivi de paramètres physico-chimiques et microbiologiques des eaux de 110 puits de la commune est réalisé afin de préciser l'importance de la pollution et d'en déterminer l'origine.

\section{MATERIEL ET METHODES}

La commune d'Abomey Calavi (Figures 1 et 2) située dans la partie sud de la République du Bénin et du département de l'Atlantique, est limitée au Nord par la commune de Zè, au Sud par l'océan Atlantique, à l'Est par les communes de SôAva et de Cotonou, et à l'Ouest par les communes de Tori-Bossito et de Ouidah (INSAE, 2003). Elle s'étend sur une superficie de $539 \mathrm{~km}^{2}$ représentant $0,48 \%$ de la superficie du Bénin (INSAE, 2003). Le climat est de type subéquatorial marqué par deux saisons pluvieuses et deux saisons sèches. La plus grande partie du territoire de la commune d'Abomey-Calavi est occupée par des sols ferrugineux tropicaux, sablonneux et 
hydromorphes très inondables (CEA-NU, 2004).

\section{Prélèvement des échantillons et évaluation des paramètres physico-chimiques}

Dans le but de mieux apprécier les caractéristiques physico-chimiques et microbiologiques des eaux de puits, notre travail a été réalisé au niveau de 110 puits qui ont été respectivement sélectionnés par tirage aléatoire simple dans l'ensemble des 55898 ménages de la commune d'Abomey-Calavi. Pour la sélection des puits, nous avons tiré au hasard trois ménages parmi ceux qui consomment les eaux de puits. Ce qui faisait au total 330 ménages utilisant l'eau des puits. Notre étude a porté sur l'eau des puits et ménages.

Pour l'enquête auprès des ménages, arrivés dans chaque localité, pour choisir le ménage à enquêter, nous avons tiré au hasard trois ménages parmi ceux qui consomment les eaux des puits sélectionnés. Dans un des trois ménages tirés, nous avons interrogé le chef de ménage ou son représentant. Lorsque dans ce ménage le chef de ménage ou son représentant a été absent, nous sommes passés dans le ménage suivant. Les investigations ont été effectuées de la manière suivante :

- Administration des questionnaires aux ménages (données sociodémographiques, approvisionnement en eau de consommation, méthode de stockage, existence de latrinespuits, gestion des ordures et des animaux, problèmes de santé liés à la consommation des eaux de puits) ;

- Observation directe des conditions de vie dans les ménages ;

- Prélèvement des eaux de puits pour les analyses en laboratoire.

Le prélèvement dans les puits a été réalisé dans des flacons stériles lestés auxquels a été fixée une ficelle permettant de les faire descendre dans le puits. L'eau a été prélevée à environ $50 \mathrm{~cm}$ de la surface libre. Les échantillons d'eau recueillis dans des flacons de $500 \mathrm{~mL}$ ont été conservés en glacière réfrigérée $\left(4^{\circ} \mathrm{C}\right)$ jusqu'au moment de l'analyse. Le suivi des paramètres physicochimiques a été effectué selon les techniques de Rodier (2009). La température, le potentiel hydrogène $(\mathrm{pH})$, la conductivité électrique (Cs) et le total des sels dissous (TDS) ont été mesurés in situ par l'analyseur multi paramétrique portatif de marque WTW 340i fournit par VWR (2008-2009). Les nitrates $\left(\mathrm{NO}_{3}{ }^{-}\right)$, les nitrites $\left(\mathrm{NO}_{2}{ }^{-}\right)$, l'azote ammoniacal $\left(\mathrm{NH}_{4}{ }^{+}\right)$, le fer total $(\mathrm{Fe})$ et l'aluminium $\left(\mathrm{Al}^{3+}\right)$ ont été déterminés par dosage colorimétrique à l'aide d'un spectrophotomètre (DR/2800). Les réactifs utilisés étaient des kits $\mathrm{HACH}$. Les réactions chimiques mises en jeu étaient les suivantes: la méthode par diazotation grâce au Nitri Ver 3 pour les nitrites, la méthode de réduction au cadmium grâce au Nitra Ver 5 pour les nitrates, la méthode au NESSLER pour l'ammonium, la méthode à la phénantroline $-1,10$ pour le fer dont la réaction consiste en une formation d'un complexe rouge orangé, la méthode aluminon grâce au réactif Alu Ver 3 pour l'aluminium. Le calcium $\left(\mathrm{Ca}^{2+}\right)$ et le magnésium $\left(\mathrm{Mg}^{2+}\right)$ ont été dosés par complexométrie à l'EDTA (Rodier, 2009) avec des limites admissibles qui sont respectivement de 100 et $50 \mathrm{mg} / \mathrm{L}$. L'oxydabilité des matières organiques (MO) a été déterminée par oxydation à chaud en milieu acide (Rodier, 2009).

L'arsenic (As) a été dosé par la spectrométrie d'absorption atomique équipé d'une lampe à cathode creuse d'arsenic avec génération d'hydrure. La méthode utilisée est celle de l'ICP-MS selon les techniques de Rodier.

Le cadmium $(\mathrm{Cd})$ et le plomb $(\mathrm{Pb})$ en présence de la flamme ont émis des photons de longueurs d'onde déterminées dont 
l'intensité a été mesurée par spectrophotométrie.

Le mercure $(\mathrm{Hg})$ a été dosé par spectrophotométrie d'absorption atomique sans flamme après minéralisation au brome. Après oxydation et minéralisation par le brome à $45^{\circ} \mathrm{C}$, le mercure a été libéré de sa solution par réduction et entraîné par un courant de gaz inerte.

Le chrome (Cr) a été dosé par spectrophotométrie d'absorption avec flamme après complexation au sel d'ammonium et d'acide pyrrolidinodithiocarboxylique.

\section{Détermination des paramètres microbiologiques}

Les paramètres microbiologiques des eaux de puits sont déterminés par des analyses bactériologique, parasitologique et mycologique.

L'analyse bactériologique a été réalisée en trois étapes :

1). La filtration sur membranes (Macy et al., 2005). Cette technique a consisté à faire passer $100 \mathrm{~mL}$ d'eau à travers une membrane cellulosique ayant des pores de diamètre uniforme égal à $0,45 \mu \mathrm{m}$; après filtration, cette membrane a été déposée dans une boîte de Pétri contenant un milieu de culture (Centre d'Expertise en Analyse Environnementale du Québec, 2000 ; Macy et al., 2005) ; des milieux de culture ont été utilisés pour la recherche des germes : le milieu ENDO pour les coliformes fermentant le lactose (Escherichia coli), le milieu Eosine Bleu de Méthylène (EMB) pour les coliformes (les entérobactéries), la Gélose de SLANETZ et BARTLEY pour les streptocoques fécaux (Centre d'Expertise en Analyse Environnementale du Québec, 2004 , 2000).

2). La numération des colonies à la loupe binoculaire après incubation à des températures de 35 à $37{ }^{\circ} \mathrm{C}$ des boîtes de Pétri ensemencés pendant 24 à 48 heures. Le dénombrement des bactéries aérobies revivifiables (germes aérobies mésophiles, hétérotrophes) visait à dénombrer non spécifiquement le plus grand nombre de micro-organismes, en particulier des bactéries se développant dans les conditions aérobies habituelles de culture. Le dénombrement de ces germes a été utilisé comme indicateur de pollution et a été également utilisé comme indicateur d'efficacité de traitement, en particulier des traitements physiques tels que la filtration qui devrait entraîner soit une très forte diminution de la concentration bactérienne par rapport à l'entrée, soit même une absence de bactéries. Ce dénombrement pouvait donner des indications importantes pour juger la validité de techniques utilisées pour la recherche d'autres paramètres. Les dénombrements ont été effectués après incubation soit à $37^{\circ} \mathrm{C}$, en 24 heures, soit à 20 ${ }^{\circ} \mathrm{C}$ en 72 heures. Sous le terme de « coliformes », a été regroupé un certain nombre d'espèces bactériennes appartenant à la famille des Enterobacteriaceae. Le dénombrement de ces organismes à $35-37{ }^{\circ} \mathrm{C}$ a été désigné sous l'expression de « dénombrement de coliformes totaux». Les coliformes comprennaient les genres: Escherichia, Citrobacter, Entérobacter, Klebsiella, Yersinia, Serratia. Les «coliformes fécaux» ou «coliformes thermo-tolérants» correspondaient à des coliformes qui présentaient les mêmes propriétés après incubation à la température de $44{ }^{\circ} \mathrm{C}$ (Macy et al., 2005).

3). L'identification des bactéries soit par la galerie API 20 (Bio Mérieux sa 69280 Marcy-L'Etoile/France) constituée de 20 microtubes prêts à l'emploi permettant de réaliser 23 tests biochimiques afin d'identifier des bacilles Gram - appartenant à la famille des Entérobacteriaceae ou non, soit par la galerie classique composée de cinq milieux de culture, à savoir le milieu Kligler, le 
milieu citrate de Simmons, le milieu mannitol mobilité, l'urée et indole (bio Mérieux sa 69280 Marcy-l'Etoile/France) et la galerie API 20 Strep pour les streptocoques fécaux.

L'analyse parasitologique a été effectuée sur le culot d'eau obtenu après centrifugation à 4000 tours/mn pendant 10 minutes de $5 \mathrm{~mL}$ de l'échantillon prélevé dans 5 L de l'échantillon séjourné après 24 heures pour la recherche éventuelle des parasites.

Enfin, l'analyse mycologique a été effectuée par la culture d'eau de puits sur la gélose sabouraud au chloramphénicol pour l'isolement, l'identification et le dénombrement des levures et moisissures.

\section{Analyses statistiques}

Les résultats obtenus sont analysés par le logiciel Stata, version 10.0. Le calcul des médianes et des pourcentages d'une part et d'autre part, des tests de comparaison de proportions (le test exact de Fisher et le test $\mathrm{du} \mathrm{Chi}^{2}$ de Pearson) a été fait. A partir d'un niveau $\mathrm{p}<0,05$, le test est retenu comme étant significatif.

\section{RESULTATS}

Les puits prélevés dans notre zone d'étude sont traditionnels, réalisés par des puisatiers locaux disposant d'un matériel rudimentaire. Ces puits sont de petits diamètres et peu profonds ( 1 à $18 \mathrm{~m}$ ). Certains de ces puits disposent de systèmes de protection, à savoir : la margelle, le couvercle.

Les résultats des analyses pratiquées sont présentés dans les Tableaux ci-dessous.

L'analyse chimique (Tableaux 1 et 2) de ces eaux de puits donnait les résultats suivants :

- $11,82 \%$ des puits investigués avaient une concentration supérieure à $45 \mathrm{mg} / \mathrm{L}$ en nitrates. Par contre les nitrites avaient été détectés dans les 110 puits prélevés avec 36 puits qui présentaient un taux supérieur à la limite acceptable;

- sur les 110 puits prélevés, 11 présentaient une concentration en aluminium supérieure à celle préconisée par la réglementation en matière de consommation et 48 avaient une concentration supérieure à la limite acceptable pour le fer total.

L'analyse des substances toxiques (Tableau 3) dans ces eaux de puits donnait les résultats ci-dessus énumérés :

$-16,36 \%, 15,45 \%$ et $14,55 \%$ des puits prélevés présentaient des taux élevés de cadmium, du plomb et du mercure. L'arsenic, avec une moyenne de $2,70 \mu \mathrm{g} / \mathrm{L}$ et le chrome total enregistraient des valeurs inférieures.

Le Tableau 4 montrait que des coliformes étaient retrouvés dans toutes les eaux et il en était de même pour Escherichia coli. Il ressort du même Tableau que 97,27\% des puits investigués présentent une pollution par les Klebsiella pneumoniae et Staphylococcus aureus, de même 96,36\% des puits prélevés sont pollués par Salmonella spp et Shigella spp. Les Streptocoques fécaux ont été identifiés dans 97 puits sur les 110 prélevés dans notre zone d'étude.

Les résultats de l'analyse parasitologique et mycologique étaient présentés dans le Tableau 5. De ce tableau, il ressortait que $29,09 \%$ et $26,36 \%$ des puits étudiés dans notre zone d'étude étaient respectivement contaminés par les trichomonas hominis et les levures.

Le Tableau 6 nous renseignait sur le pourcentage de diarrhées suivant les paramètres ménage à Abomey-Calavi en 2009.

Les résultats de ce tableau montraient que :

- 80,56\% de ceux qui avaient construit leurs latrines à moins de $15 \mathrm{~m}$ de leurs puits faisaient les diarrhées alors que c'était le cas de $19,44 \%$ des sujets de l'autre groupe avec 
une différence statistiquement significative $(\mathrm{p}=0,005)$;

- 92\% de ceux qui n'utilisaient pas des latrines dans leurs ménages faisaient les diarrhées alors que c'était le cas de $8 \%$ des sujets de l'autre groupe avec une différence statistiquement significative ( $\mathrm{p}=0,011)$;

- $64,10 \%$ de ceux qui ne couvraient pas leurs puits faisaient les diarrhées alors que c'était le cas de 35,90\% des sujets de l'autre groupe avec une différence statistiquement significative $(\mathrm{p}=0,005)$;

$-91,43 \%, 81,82 \%, 90 \%, 60 \%$ et $29,63 \%$ de ceux qui étaient respectivement non scolarisé, de niveau primaire, secondaire, professionnel et supérieur faisaient les diarrhées alors que c'était le cas de $8,57 \%, 18,18 \%, 10 \%, 40 \%$ et $70,37 \%$ respectivement des sujets de l'autre groupe avec une différence statistiquement significative $(\mathrm{P}<0,001)$.

Tableau 1 : Résultats de l'analyse physico-chimique liée à la structure naturelle des eaux de puits et aux substances chimiques indésirables à Abomey-Calavi en 2009.

\begin{tabular}{lccc}
\hline Paramètres & Limite acceptable & n & Me (P25 - P75) \\
\hline Température ${ }^{\circ} \mathrm{C}$ & 25 & 110 & $29(28,5-29,9)$ \\
Conductivité $\mu \mathrm{S} / \mathrm{cm}$ & 2000 & 110 & $144,55(85,2-227)$ \\
Total des sels dissous (TDS) $\mathrm{mg} / \mathrm{L}$ & 2000 & 110 & $111(58-176)$ \\
Ammonium $\mathrm{mg} / \mathrm{L}$ & 0,5 & 110 & $0,07(0,04-0,14)$ \\
Oxydabilité $\mathrm{mgO} / \mathrm{L}$ & 5 & 110 & $2,3(2,07-2,4)$ \\
Calcium $\mathrm{mg} / \mathrm{L}$ & 100 & 110 & $5,11(3,61-7)$ \\
Magnesium mg/L & 50 & 110 & $2,74(2,2-4,2)$ \\
\hline
\end{tabular}

$\mathrm{n}$ : taille de l'échantillon, Me : Médiane, P25 : Percentile 25, P75 : Percentile 75.

Tableau 2 : Résultats de l'analyse physico-chimique liée à la structure naturelle des eaux de puits non conformes et des substances chimiques indésirables à Abomey-Calavi en 2009.

\begin{tabular}{lcccc}
\hline Paramètres & & Limite acceptable & n & Pourcentage \\
\hline $\mathrm{pH}$ Unité $\mathrm{pH}$ & $<6,5$ & & 108 & 98,2 \\
& $>=6,5$ & $6,5-8,5$ & 02 & 1,8 \\
& $<=0,2$ & & 99 & 90 \\
Aluminium mg/L & $>0,2$ & 0,2 & 11 & 10 \\
& $<=45$ & & 97 & 88,18 \\
Nitrates mg/L & $>45$ & 45 & 13 & 11,82 \\
& $<=0,1$ & & 74 & 67,27 \\
Nitrites mg/L & $>0,1$ & 0,1 & 36 & 32,73 \\
& $\leq 0,3$ & & 62 & 56,36 \\
Fer mg/L & $>0,3$ & 0,3 & 48 & 43,64 \\
& $\leq 3$ & & 102 & 92,73 \\
DBO5 mgO2/L & $>3$ & 3 & 08 & 7,27 \\
\hline
\end{tabular}


Tableau 3 : Résultats de l'analyse des substances toxiques des eaux de puits à Abomey-Calavi en 2009.

\begin{tabular}{lccccc}
\hline Paramètres & & Limite acceptable & n & Moy (DS) & Pourcentage \\
\hline Arsenic $\mu \mathrm{g} / \mathrm{L}$ & $\leq 5$ & 50 & 110 & $2,70(0,87)$ & \\
& $>5$ & 5 & 92 & & 83,64 \\
Cadmium $\mu \mathrm{g} / \mathrm{L}$ & $\leq 1$ & & 18 & & 16,36 \\
& $>1$ & 1 & 94 & & 85,45 \\
Mercure $\mu \mathrm{g} / \mathrm{L}$ & $\leq 50$ & & 16 & 14,55 \\
& $>50$ & 50 & 93 & 84,55 \\
Plomb $\mu \mathrm{g} / \mathrm{L}$ & 0,05 & 17 & 15,45 \\
Chrome total mg/L & \multicolumn{5}{c}{100} \\
\hline n: taille de l'échantillon, Moy : Moyenne, DS : Déviation Standard.
\end{tabular}

Tableau 4 : Répartition des puits selon les germes identifiés à Abomey-Calavi en 2009.

\begin{tabular}{lcc}
\hline Germes identifiés & Nombre de puits Investigués & Pourcentage \\
\hline Coliformes & 110 & 100 \\
Escherichia Coli & 110 & 100 \\
Streptocoques fécaux & 97 & 88,18 \\
Clostridium perfringens & 105 & 95,45 \\
Salmonella spp & 106 & 96,36 \\
Shigella spp & 106 & 96,36 \\
Klebsiella pneumoniae & 107 & 97,27 \\
Pseudomonas aeruginosa & 104 & 94,55 \\
Staphylococcus aureus & 107 & 97,27 \\
Candida albicans & 34 & 30,91 \\
\hline
\end{tabular}

Tableau 5 : Résultats de l'analyse parasitologique et mycologique des eaux de puits à AbomeyCalavi en 2009.

\begin{tabular}{lccc}
\hline Paramètres & Absence/Présence & Fréquence & Pourcentage \\
\hline Trichomonas hominis & Absence & 78 & 70,91 \\
& Présence & 32 & 29,09 \\
\hline Kyste Entamoeba coli & Absence & 106 & 96,36 \\
& Présence & 04 & 3,64 \\
\hline Kyste Entamoeba & Absence & 109 & 99 \\
histolytica & Présence & 01 & 01 \\
\hline Kyste de Giardia & Absence & 106 & 96,4 \\
intestinalis & Présence & 04 & 3,6 \\
\hline Ankylostoma duodenale & Absence & 103 & 93,64 \\
& Présence & 07 & 6,36 \\
\hline Levures & Absence & 81 & 73,64 \\
& Présence & 29 & 26,36 \\
\hline
\end{tabular}


Tableau 6 : Pourcentage de diarrhées suivant les paramètres ménage à Abomey-Calavi en 2009.

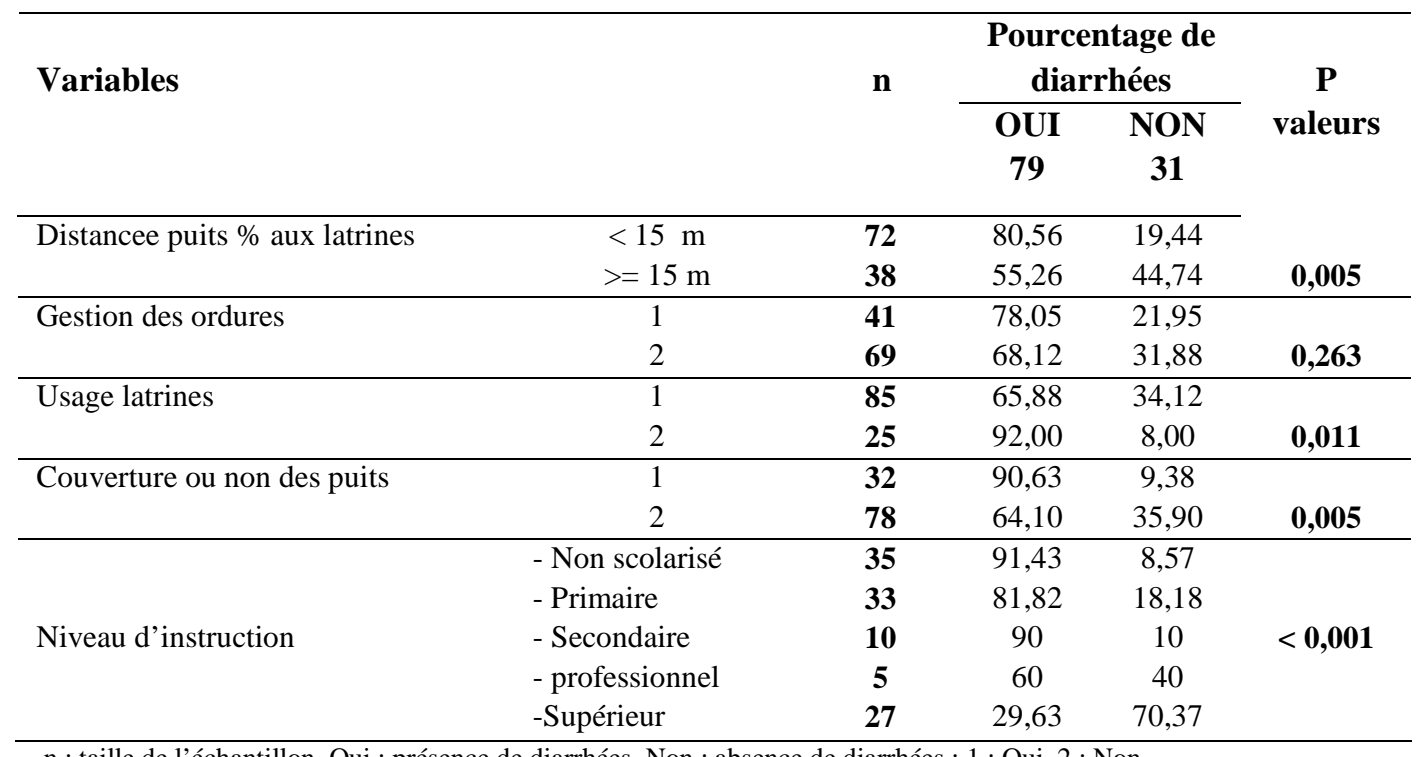

$\mathrm{n}$ : taille de l'échantillon, Oui : présence de diarrhées, Non : absence de diarrhées ; 1 : Oui, 2 : Non.

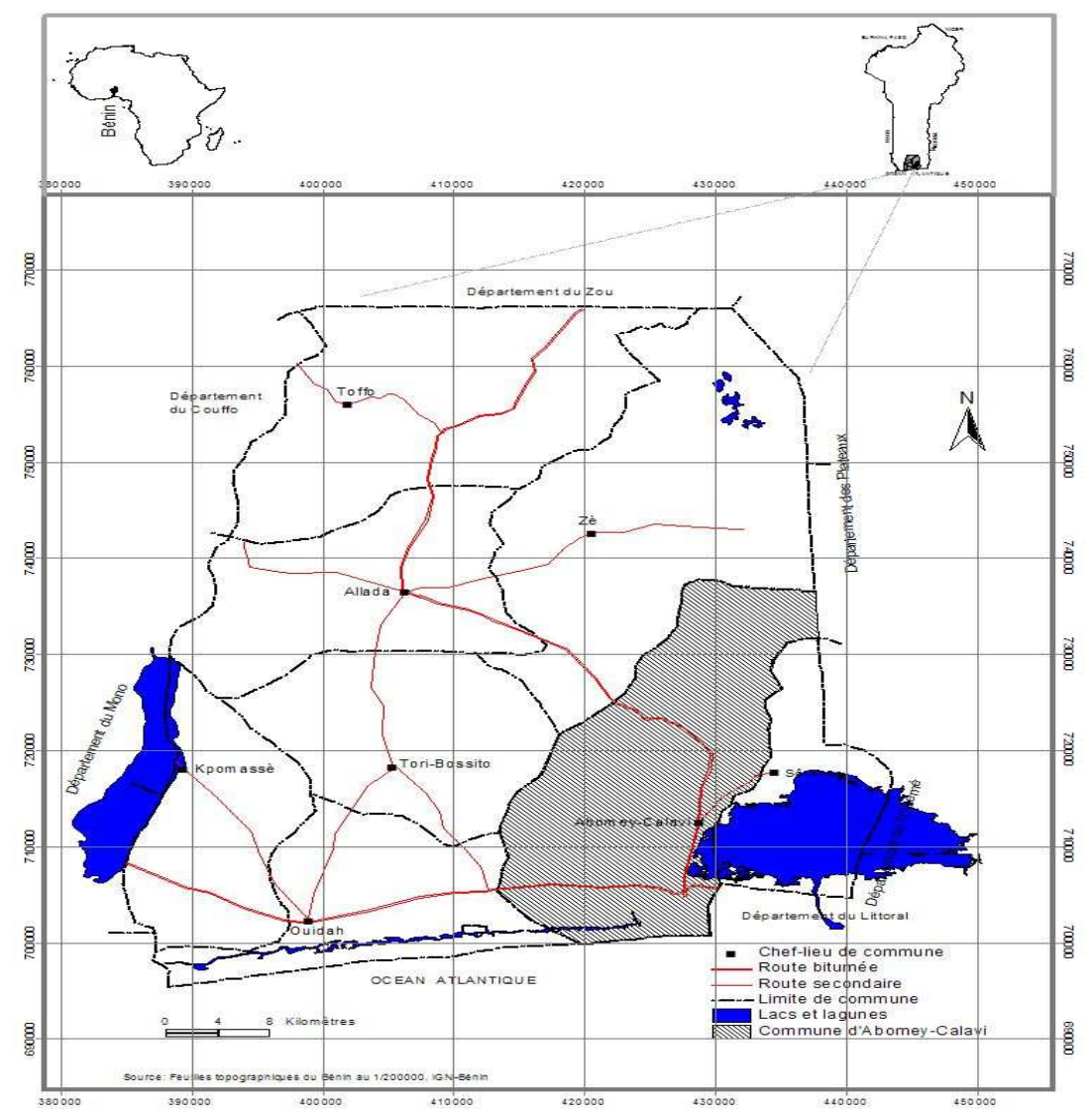

Figure 1: Carte du département de l'Atlantique situant la commune d'Abomey-Calavi au Bénin. Source : Feuille topographique du Bénin au 1/200000 IGN -Bénin. 


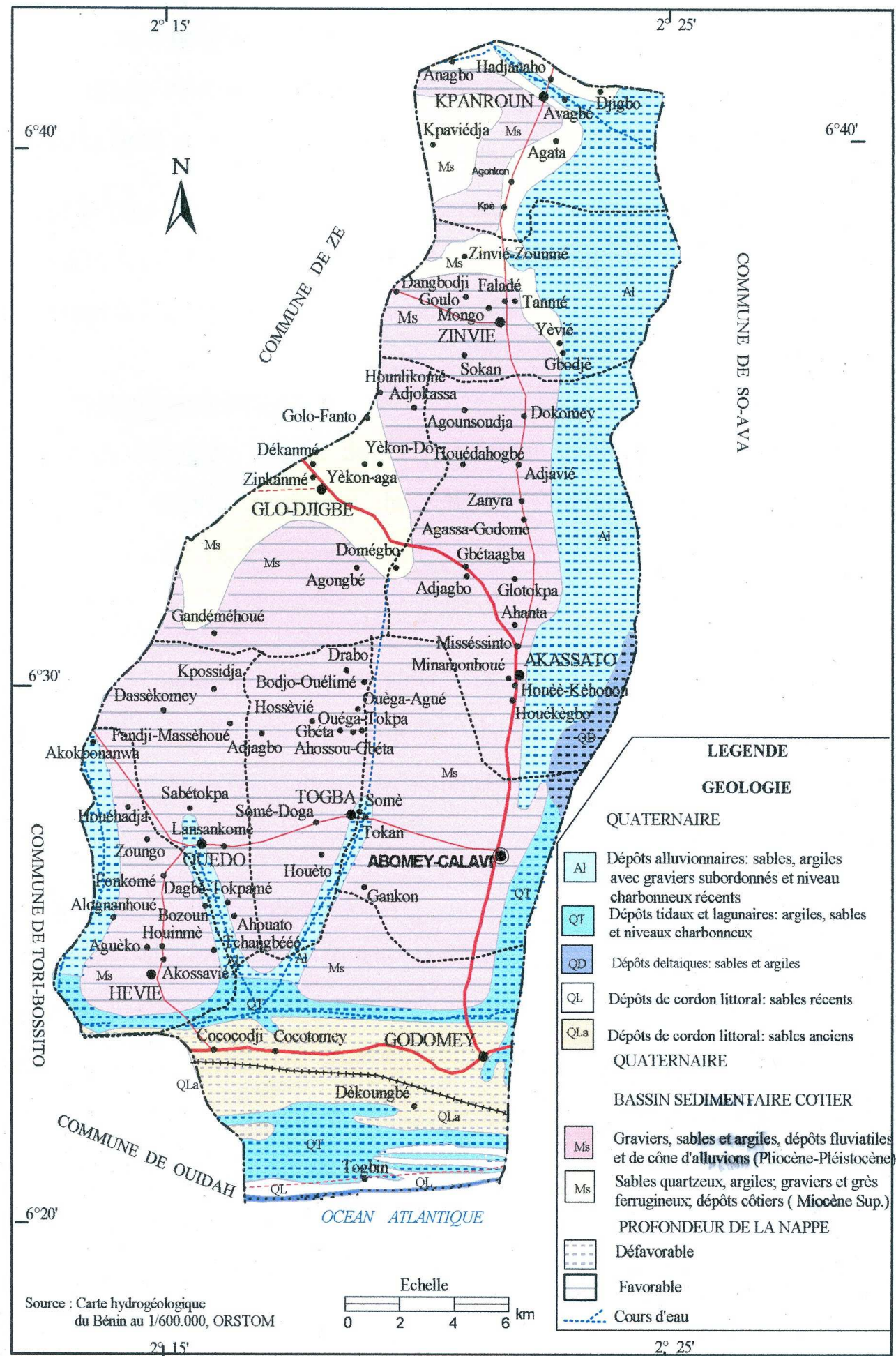

Figure 2: Carte la commune d'Abomey-Calavi situant l'hydrogéologie. Source : Carte hydrogéologique du Bénin au 1/600000 ORSTOM. 


\section{DISCUSSION}

Plus de $70 \%$ de la population de la commune d'Abomey-Calavi (307.745 habitants) est actuellement approvisionnée en eau par les puits et forages des nappes souterraines (PNE, 2006). La protection de la qualité de ces eaux est donc un objectif prioritaire.

Les résultats de l'analyse physicochimique présentés dans ce travail ont montré que la conductivité, l'ammonium, l'oxydabilité, le calcium, le magnésium, le total des sels dissous, peuvent être considérés admissibles et ne présentent aucune incidence sur la qualité de l'eau des puits. Ces résultats sont en accord avec ceux obtenus par Chippaux et al. (2002) au Niger et Saâdia et al. (2007) au Maroc. Les résultats des essais physico-chimiques ont révélé également l'existence d'une température élevée sur l'ensemble des puits étudiés avec une moyenne de $29,23{ }^{\circ} \mathrm{C}$ supérieure à la limite acceptable qui est de $25{ }^{\circ} \mathrm{C}$ recommandée par l'OMS. Ces températures élevées pourraient s'expliquer par l'influence de la chaleur ambiante sur les eaux prélevées et aussi par le gradient géothermique de la zone. Les résultats de notre étude montrent que 98,2\% des puits prélevés ont un $\mathrm{pH}$ en dessous de la normale qui est de 6,5 et 8,5 unités $\mathrm{pH}$, ce qui pourrait expliquer un risque de corrosion du ciment ou des métaux des canalisations avec entraînement de plomb. L'acidité du $\mathrm{pH}$ au niveau des puits dans notre étude est semblable à l'étude faite par Nduka et al. (2007) à Warri au Nigeria. Les résultats de l'analyse physico-chimique montrent que $10 \%$ et $43,64 \%$ des puits ont un taux d'aluminium et de fer total respectivement supérieur à la limite admissible qui est de $0,2 \mathrm{mg} / \mathrm{L}$ et 0,3 $\mathrm{mg} / \mathrm{L}$. Les eaux de puits étudiés affichent également une concentration élevée en nitrates et en nitrites. Les nitrates $\left(\mathrm{NO}_{3}{ }^{-}\right)$et les nitrites $\left(\mathrm{NO}_{2}{ }^{-}\right)$sont des ions présents de façon naturelle dans l'environnement. Ils sont le résultat d'une nitrification de l'ion ammonium $\left(\mathrm{NH}_{4}^{+}\right)$, présent dans l'eau et le sol, qui est oxydé en nitrites par les bactéries du genre
Nitrosomonas, puis en nitrates par les bactéries du genre Nitrobacter. Les nitrates sont très solubles dans l'eau, ils migrent donc aisément dans la nappe phréatique lorsque les niveaux excèdent les besoins de la végétation. La toxicité des nitrates résulte de leur réduction en nitrites et de la formation de méthémoglobine d'une part et de leur contribution possible à la synthèse endogène de composés N-nitrosés d'autre part. La présence de nitrates dans l'eau de consommation est principalement attribuable aux activités humaines (Saâdia et al., 2007). Une eau qui renferme des nitrites est considérée comme suspecte car lui est souvent associée une détérioration de la qualité microbiologique. Dans notre milieu d'étude, l'augmentation des teneurs en nitrates constatées est notamment liée aux activités humaines et aux pratiques agricoles intensives. Les installations septiques déficientes, de même que la décomposition de la matière végétale et animale, peuvent aussi être une source de nitrates dans l'eau. Le risque de contamination est plus important si le sol recouvrant la nappe d'eau est vulnérable et si la nappe est peu profonde (puits de surface).

La méthémoglobinémie du nourrisson est le seul effet sur la santé qui a été associé de façon non équivoque à une exposition excessive aux nitrates par l'eau de consommation. La méthémoglobinémie résulte de la réduction des nitrates en nitrites par les microorganismes du système digestif, suivie de l'oxydation par les nitrites du fer ferreux $\left(\mathrm{Fe}^{2+}\right)$ de l'hémoglobine en fer ferrique $\left(\mathrm{Fe}^{3+}\right)$, qui engendre la méthémoglobine. La méthémoglobine, contrairement à l'hémoglobine, est incapable de fixer l'oxygène, ce qui contribue à réduire le transport de l'oxygène des poumons vers les tissus. La formation des nitrites pourrait parfois résulter d'une contamination bactérienne de l'eau, ayant pour effet de réduire les nitrates en nitrites avant même qu'ils ne soient ingérés. Ces valeurs élevées en nitrates et nitrites sont rapportées par 
Dégbey et al. (2008) qui ont travaillé sur la qualité de l'eau de puits dans la commune d'Abomey-Calavi au Bénin. La pollution nitrique des eaux souterraines étudiées est due à l'épandage de fumier ou d'engrais chimiques, de même que des installations septiques avoisinantes. La variation de la concentration en nitrates constatée entre les différents puits peut être liée entre autres à l'hétérogénéité du milieu physique. Les nitrates sont naturellement présents mais en faible quantité dans les sols. Ils constituent aujourd'hui le principal indicateur d'une dégradation des ressources en eau. L'augmentation des teneurs constatées depuis quelques années est notamment le résultat d'activités humaines, comme les pratiques agricoles intensives. La consommation de cette eau devrait être interdite aux femmes enceintes et aux nourrissons pour prévenir la méthémoglobinémie.

Les métaux lourds sont des polluants engendrés par l'activité humaine qui ont un fort impact toxicologique (Azeez et al., 2009). Les métaux toxiques sont nombreux, mais on peut citer surtout l'arsenic, le cadmium, le plomb et le mercure. Ils ont des impacts sur les produits de consommation courante et sur l'homme ( Nduka et al., 2008). Il est assez difficile de prévoir l'évolution des métaux dans l'environnement, car ils peuvent subir un grand nombre de transformations (oxydation, réduction, complexation, etc.), et cette évolution dépend fortement du milieu (Azeez et al., 2009). En effet, la migration des métaux lourds vers la nappe phréatique est fonction de nombreux paramètres: la forme chimique initiale du métal ; la perméabilité du sol et du sous-sol ; la porosité du sol ; le pH; l'activité biologique; le potentiel redox du sol; la composition minéralogique du sol; la teneur en matières organiques du sol. Les principales sources de contamination de l'eau sont les suivantes: les eaux usées domestiques et industrielles, la production agricole, les polluants atmosphériques, les anciennes décharges, l'utilisation de substances dangereuses pour l'eau, etc. Même si les métaux lourds sont le plus souvent présents à l'état de trace, ils n'en restent pas moins très dangereux, puisque leur toxicité se développe par bioaccumulation dans les organismes. Une exposition de courte durée (quelques jours ou semaines) au cadmium présent dans l'eau de consommation à des concentrations élevées peut causer des nausées, des vomissements et de la diarrhée. Une exposition à long terme (plusieurs années ou décennies) au cadmium présent dans l'eau de consommation peut causer des dommages aux reins. Le saturnisme provoqué par un excès de plomb se traduit par des troubles cliniques, des anomalies biologiques et des altérations histopathologiques variées. Il est influencé par de multiples facteurs et principalement par l'action du plomb comme inhibiteur enzymatique. Pour le mercure, son intoxication par les sels mercuriques se traduit par une stomatite, des troubles neurologiques, un syndrome néphrotique. Le mercure peut aussi donner des dérivés organomercuriels de toxicité nettement plus élevée. Le mécanisme de l'action toxique pourrait être lié à une interaction avec les groupements thiols des protéines; par ailleurs, le méthylmercure inactiverait de nombreux systèmes enzymatiques.

Les puits de la commune connaissent de fortes pollutions en indicateurs de contamination fécale, en accord avec ceux trouvées par Boutin et Dias (1987) pour la nappe de Marrakech au Maroc, par Bordalo et Savva (2007) au cours de la recherche pour l'eau potable sûre en Guinée Bissau et enfin par Egwri et Aboaba (2002) à Lagos au Nigeria sur l'Impact environnemental de la qualité bactériologique de l'approvisionnement en eau domestique. La majorité des microorganismes pathogènes susceptibles de se trouver dans l'eau proviennent de déjections humaines ou animales. Les bactéries $E$. coli font partie du groupe des coliformes totaux. C'est une espèce très abondante dans la flore intestinale humaine et animale, et c'est aussi la seule qui soit strictement d'origine fécale. Les sources 
locales de contamination peuvent être multiples : aménagement du puits (manque d'étanchéité du couvercle ou du scellement), sol environnant (absence d'un monticule autour du puits pour éloigner le ruissellement de surface), installation septique défectueuse ou encore insalubrité des lieux (ex., épandage de fumier ou autres activités générant de la pollution fécale). Il s'agit donc dans ces cas de procéder aux travaux requis pour corriger la situation. Des analyses subséquentes dans le cadre du suivi de la qualité de l'eau permettront de vérifier l'efficacité des correctifs apportés. Des résultats d'analyses ponctuelles conformes ne peuvent assurer à eux seuls la qualité de l'eau de boisson en tout temps. Il faut donc prendre les précautions nécessaires pour assurer la salubrité et le bon fonctionnement d'un système individuel d'approvisionnement en eau de boisson (Gundry et al., 2009). À l'instar des précautions prises à l'égard du puits, des mesures de prévention doivent également être appliquées aux systèmes de traitement pour l'élimination des problèmes esthétiques (Kreamer et Usher, 2009). Un manquement à ces mesures de prudence pourrait favoriser la prolifération des bactéries. Pour assurer une désinfection efficace, l'eau qui alimente les systèmes de traitement pour l'élimination des microorganismes doit être claire et limpide (Gundry et al., 2009).

Les forts taux de pourcentage en coliformes et streptocoques fécaux au niveau des puits de la commune sont similaires à ceux trouvées par Nola et al. (1998) au niveau de la nappe phréatique de Yaoundé au Cameroun. De même, des forts taux en coliformes fécaux sont enregistrés au niveau des eaux de puits de la nappe phréatique de Yembeul au Sénégal par Tandia et al. (1997). En effet, le fort taux en coliformes totaux et en streptocoques fécaux enregistré au niveau des puits peut être attribué aux mauvaises conditions d'hygiène enregistrées dans la zone d'étude. Par ailleurs, les eaux superficielles chargées en microorganismes s'infiltrant dans le sol sablonneux, parviennent à la nappe sans avoir bénéficié d'une filtration efficace, et occasionnent une multitude de pollutions ponctuelles. La pollution bactériologique de l'eau de puits est plus inquiétante car elle pourrait signifier une insalubrité générale des eaux souterraines de la ville. Les nappes superficielles, accessibles par les puits de la commune d'Abomey-Calavi au Bénin, apparaissent fortement contaminées par des matières organiques d'origine humaines et animales comme c'est le cas d'une étude faite au Burkina Faso par Poda et al. (2003).

La qualité microbiologique est une préoccupation sanitaire essentielle. L'eau consommée doit être indemne d'organismes pathogènes. Le risque à court terme peut se traduire par des troubles intestinaux dont l'apparition et l'importance dépendent de l'état général de santé du consommateur et de sa sensibilité. Sur la plan bactériologique, on note une présence massive des streptocoques et coliformes fécaux dans ces eaux quelles que soient les sources à Abomey-calavi et dans d'autres pays par Lévesque et al. (2008). Les impacts sanitaires de ce phénomène sont la forte prévalence des diarrhées et des parasitoses intestinales chez les enfants de moins de 5 ans, les femmes enceintes et les personnes de troisième âge dans l'écosystème étudié. Dans notre étude, les diarrhées endémiques sont associées à la contamination fécale de l'eau des puits qui constituent de grands risques pour le consommateur. C'est aussi le constat fait par Gundry et al. (2009) dans leur région.

L'analyse parasitologique qualitative et mycologique a révélé la présence dans l'eau des kystes d'Entamoeba histolytica, coli de Giardia intestinalis, des œufs et larves d'Ancylostoma duodenale, puis des levures qui peuvent être à l'origine des diarrhées, des gastro-entérites, de la dysenterie amibienne et des mycoses.

En effet, dans cette commune, la population ne dispose pas d'un système de traitement des ordures ménagères, encore moins d'un système de collecte, de traitement et d'évacuation des eaux usées. Quant aux 
latrines, 80,56 \% sont situées à une distance non conforme à la norme minimale recommandée par l'OMS qui est de $15 \mathrm{~m}$ entre latrines et puits. La mauvaise gestion des ordures ménagères et des eaux usées, la contamination du sol par les excréta humains, le non-respect de la distance entre les latrines et les puits se traduisent par la forte teneur de l'eau en certains éléments chimiques (nitrites, nitrates, la demande biologique en oxygène en 5 jours) qui sont des indices de pollution. Cette pollution chimique observée à l'issue de l'analyse de certaines eaux de puits prélevées dans notre série d'étude est conforme aux conclusions de Dégbey et al. (2008) dans la commune d'Abomey-Calavi au Bénin. Les résultats de l'analyse bactériologique des eaux ont révélé que la totalité des puits prélevés étaient contaminés.

\section{Conclusion}

Les résultats de l'analyse physicochimique de l'eau de puits dans la commune d'Abomey-Calavi au Bénin ont montré que la conductivité, l'oxygène dissous, l'ammonium, l'oxydabilité, le calcium, le magnésium et le total des sels dissous, peuvent être considérés admissibles et ne présentent aucun danger pour la consommation. Par contre le $\mathrm{pH}$ souvent trop bas peut présenter un problème de solubilisation de différents métaux toxiques (plomb, cadmium, mercure ; etc.). Les eaux de puits étudiés affichent des concentrations en nitrates et nitrites respectivement supérieures aux normes de l'O.M.S. Ces résultats confirment l'impact de l'intensification de l'agriculture, les rejets des eaux usées domestiques et industriels. Il est à signaler que les puits de la commune affichent des concentrations élevées en aluminium et en fer total. La présence très élevée des germes indicateurs de la contamination fécale, ainsi que la présence d'autres germes responsables d'infections transmises par l'eau, constituent sans doute une menace pour les habitants qui tirent l'eau nécessaire à la majeure partie de leurs besoins à partir de l'eau de ces puits. Les études doivent se poursuivre pour surveiller l'évolution de la pollution de la nappe, notamment en profondeur.

\section{REMERCIEMENTS}

Ce travail a été réalisé avec l'appui de la Coopération Technique Belge (CTB). Nous exprimons notre gratitude au personnel du laboratoire de contrôle de qualité des eaux et aliments du ministère de la santé pour leur soutien constant lors de l'élaboration et la réalisation de ce travail. Nous remercions également les autorités de la commune d'Abomey-Calavi pour avoir accepté la réalisation de cette étude.

\section{REFERENCES}

Azeez JO, Adekunle IO, Atiku OO, Akande KB, Jamiu-azeez SO. 2009. Effect of nine years of animal waste deposition on profile distribution of heavy metals in Abeokuta, south-western Nigeria and its implication for environmental quality. Waste. Manag., 29(9): 2582-6.

Bordalo AA, Savva-bordalo J. 2007. The quest for safe drinking water: an example from Guinea-Bissau (West Africa). Water. Res., 41(13): 2978-86.

Bourne LT, Harmse B, Temple N. 2007. Water: a neglected nutrient in the young child? A South African perspective. Matern. Child. Nutr. , 3(4):303-11.

Boutin C, Dias N. 1987. Impact de l'épandage des eaux usées de la ville de Marrakech sur la nappe phréatique. Bull. Fac. Sci. Marrakech (Sect. Sci. Vie), 3: 5-27.

Centre d'Expertise en Analyse Environnementale du Québec. 2000. Recherche et Dénombrement des Coliformes Totaux ; Méthode par Filtration sur Membrane. Gouvernement du Québec : Québec.

Centre d'Expertise en Analyse Environnementale du Québec. 2000. Recherche et Dénombrement des Coliformes Fécaux ; Méthode par Filtration sur Membrane. Gouvernement du Québec : Québec. 
Centre d'Expertise en Analyse Environnementale du Québec. 2004. Recherche et Dénombrement des Entérocoques : Méthode par Filtration sur Membrane. Gouvernement du Québec : Québec:

Chippaux JP, Houssier S, Gross P, Bouvier C, Brissaud F. 2002. Etude de la pollution de l'eau souterraine de la ville de Niamey, Niger. Bull Soc. Pathol. Exot., 95(2): 119123.

Commission Economique des Nations Unies pour l'Afrique. 2004. Aquifère Superficiel et Pollution Urbaine en Afrique de l'Ouest. Cotonou (Bénin). CEA-NU, Rapport UNESCO Cotonou.

Dégbey C, Makoutodé M, Ouendo EM, Fayomi B, De brouwer C. 2008. La qualité de l'eau de puits dans la commune d'Abomey-Calavi au Bénin. Environnement. Risques. Santé, 7(4): 279283

Egwari L, Aboaba OO. 2002. Environmental impact on the bacteriological quality of domestic water supplies in Lagos, Nigeria. Rev. Saude. Publica., 36(4): 513-20.

Gundry SW, Wright JA, Conroy RM, Preez MD, Genthe B, Moyo S Mutisi C, Potgieter N . 2009. Child dysentery in the Limpopo Valley: a cohort study of water, sanitation and hygiene risk factors. $J$. Water. Health. , 7(2): 259-66.

Institut National de la Statistique et de l'Analyse Economique. 2003. Troisième recensement général de la population et de l'habitation, résultats définitifs: caractéristiques générales de la population. INSAE: Cotonou.

Kreamer DK, Usher B. 2009. Sub-Saharan African Ground Water ProtectionBuilding on International Experience .Ground.Water. ,48(2): 257-268.

Landreau.1990. La pollution des eaux par les nitrates. Eau.Développement., 2(10): 4958

Levesque B, Pereg D, Watkinson E, Maguire JS, Bissonnette L, Gingras S, Rouja P,
Bergeron MG, Dewailly E. 2008. Assessment of microbiological quality of drinking water from household tanks in Bermuda. Can. J.Microbiol. , 54: 495500

Macy JT, Dunne EF, Angoran-benie YH, Kamelan-tano Y, Kouadio L, Djai KA, Luby SP. 2005. Comparison of two methods for evaluating the quality of stored drinking water in Abidjan, Côte d'lvoire, and review of other comparisons in the literature. J. Water. Health., 3(3): 221-8.

Ministère de la Santé. Direction de la Programmation et de la Prospective. 2009. Service des statistiques et de la documentation. Annuaire des statistiques sanitaires. Ministère de la santé : Cotonou.

Nduka JK, Orisakwe OE, Ezenweke LO, Chendo MN , Ezenwa TE .2008. Heavy metal contamination of foods by refuse dump sites in Awka, southeastern Nigeria. Scientific. World .Journal. , 8: 941-8.

Nduka JK, Orisakwe OE. 2007. Heavy metal levels and physico--chemical quality of potable water supply in Warri, Nigeria. Ann. Chim. , 97(9): 867-74.

Monkiedje S, Foto M. 2006. Transfert des bactéries fécales vers une nappe phréatique à travers une colonne de sol en région équatoriale : influence de la charge en eau appliquée en surface. Rev. Sci. Eau., 19(2): 101-112.

Nola M, Njine T, Monkiedje A, SIkati FV, Djuikom E. 1998. Qualité bactériologique des eaux des sources et des puits de Yaoundé (Cameroun). Cahiers. Santé, 8(5): 330-336.

Organisation Mondiale de la Santé.1994. Directives de Qualité pour l'Eau de Boisson: Recommandation. OMS : Genève.

Organisation Mondiale de la Santé .2003. L'eau pour les hommes, l'eau pour la vie. OMS, UNESCO-WWAP : Paris.

Pande S, Keyzer MA, Arouna A, Sonneveld BG. 2008. Addressing diarrhea prevalence in the West African Middle Belt: social 
and geographic dimensions in a case study for Benin. Int. J .Health. Géogr., 7: 17.

Partenariat National de l'Eau. 2006. Livre Bleu, l'Eau, l'Assainissement, la Vie et le Développement Humain Durable. PNE: Bénin.

Poda JN, Gagliardi R, Kam FO, Niameogo AT. 2003. La perception des populations des maladies diarrhéiques au Burkina Faso: une piste pour l'éducation aux problèmes de santé. Vertigo -Revue. Electronique. Sciences .Environnement, 4(1): 1-6.

Rodier J. 2009. L'analyse de l'Eau: Eaux Naturelles, Eaux Résiduaires, Eau de Mer:
Physico-chimie, Bactériologie et Biologie (9ème édn). Dunod : Paris; 1383p.

Saadia B, Khadija O, Saïd O, Nourredine EH, Benaissa A .2007. Etude de la qualité physico-chimique et bactériologique de la nappe phréatique M'nasra (Maroc). Afrique. Science, 03(3): 391-404.

Tandia AA, Gaye CB, Faye A. 1997. Origine des teneurs élevées en nitrates dans la nappe phréatique des sables quaternaires (région de Dakar, Sénégal).Sécheresse. 8(4): 291-4. 